\title{
Aspects of planar, oblique and interacting shock waves in an ideal dissociating gas
}

\author{
S. R. Sanderson, ${ }^{\text {a) }}$ H. G. Hornung, and B. Sturtevant ${ }^{\text {b) }}$ \\ Graduate Aeronautical Laboratories, California Institute of Technology, Pasadena, California 91125
}

(Received 11 June 2002; accepted 14 March 2003; published 5 May 2003)

\begin{abstract}
We develop a compact dimensionless framework for the analysis of canonical thermo-chemical nonequilibrium flow fields involving normal, oblique and interacting shock waves. Discontinuous solutions of the conservation equations are coupled with thermodynamic and kinetic models for an ideal dissociating gas. Convenient forms are provided for the variation of the relevant dimensionless parameters across shock waves in dissociating gases. The treatment is carried through in a consistent manner for the pressure-flow deflection angle plane representation of shock wave interaction problems. The contribution of the current paper is a careful nondimensionalization of the problem that yields a tractable formulation and allows results with considerable generality to be obtained.
\end{abstract}

(C) 2003 American Institute of Physics. [DOI: 10.1063/1.1572162]

\section{INTRODUCTION}

Our objective in the current paper is to develop a consistent set of dimensionless forms for the analytical treatment of normal, oblique and interacting shock waves in a chemical nonequilibrium dissociating gas. The model that is described here begins with the usual conservation equation analysis of shock waves. Normal shock wave solutions are obtained in the chemically nonreacting (or frozen) limit and the thermochemical equilibrium limit using simple thermodynamic and kinetic models. The rate at which the dissociation reaction proceeds from the frozen state to the equilibrium state is described by standard Arrhenius rate expressions. The extension from normal to oblique shock waves is obtained by the conventional technique of applying the one-dimensional solutions to the component of velocity normal to the shock front. To facilitate the computation of multiple shock jumps in the solution of interaction problems we develop a convenient means of determining the downstream dimensionless parameters, that become the upstream parameters for subsequent shock waves. With this framework established, it is a simple matter to elucidate the essential behavior of simple shock wave flow fields and to develop the working detail required to map chemical nonequilibrium shock wave interaction problems into the pressure-flow deflection angle plane. The disparate shock strengths produced at the shock wave intersection point in Mach reflection stand out as a profitable avenue for further investigation.

\section{NORMAL SHOCK FOR AN IDEAL DISSOCIATING GAS}

We begin by deriving the jump conditions for a normal shock wave in a relaxing gas. The problem is known in the literature (e.g., Vincenti and Kruger ${ }^{1}$ ). Here we propose a

\footnotetext{
${ }^{a)}$ Present address: GE Global Research Center, Bldg. K1, P.O. Box 8, Schenectady, New York 12301.

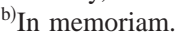

compact dimensionless form and allow for the nonequilibrium upstream state required for the analysis of multiple wave interaction problems. Consider the relaxation to equilibrium of the system,

$$
\mathrm{N}_{2}+\mathrm{M} \rightleftharpoons \mathrm{N}+\mathrm{N}+\mathrm{M},
$$

where here nitrogen is the prototypical gas and $\mathrm{M}$ represents a nonreacting third body. The translational and rotational shock thickness is neglected and we consider only the relaxation of the internal modes over a length scale comparable to that of the fluid motion (see Fig. 1). In the following the translational/rotational shock is referred to as the translational discontinuity for simplicity. The mass, momentum and energy conservation equations that apply across the onedimensional translational discontinuity and throughout the downstream relaxation zone are

$$
\begin{aligned}
& \rho_{2} u_{2}=\rho_{1} u_{1}, \\
& p_{2}+\rho_{2} u_{2}^{2}=p_{1}+\rho_{1} u_{1}^{2}, \\
& h_{2}+\frac{1}{2} u_{2}^{2}=h_{1}+\frac{1}{2} u_{1}^{2},
\end{aligned}
$$

where $\rho$ is the density of the gas, $u$ is the velocity normal to the shock, $p$ is the pressure and $h$ is the enthalpy per unit mass. The subscripts, 1 and 2, refer here to the upstream and downstream states, respectively. In dimensionless form Eqs. (2) and (3) become

$$
\begin{aligned}
& \hat{\rho} \hat{u}=1, \\
& \hat{p}=1+\frac{1}{P_{1}} \frac{\hat{\rho}-1}{\hat{\rho}},
\end{aligned}
$$

where the variables are normalized with respect to their upstream values and generally the notation $\hat{\phi}=\phi_{2} / \phi_{1}$ applies. The dimensionless parameter, $P_{1}$, arises and it behaves as $P_{1} \sim 1 / M_{1}^{2}$ for a perfect gas:

$$
P_{1}=p_{1} / \rho_{1} u_{1}^{2} \text {. }
$$




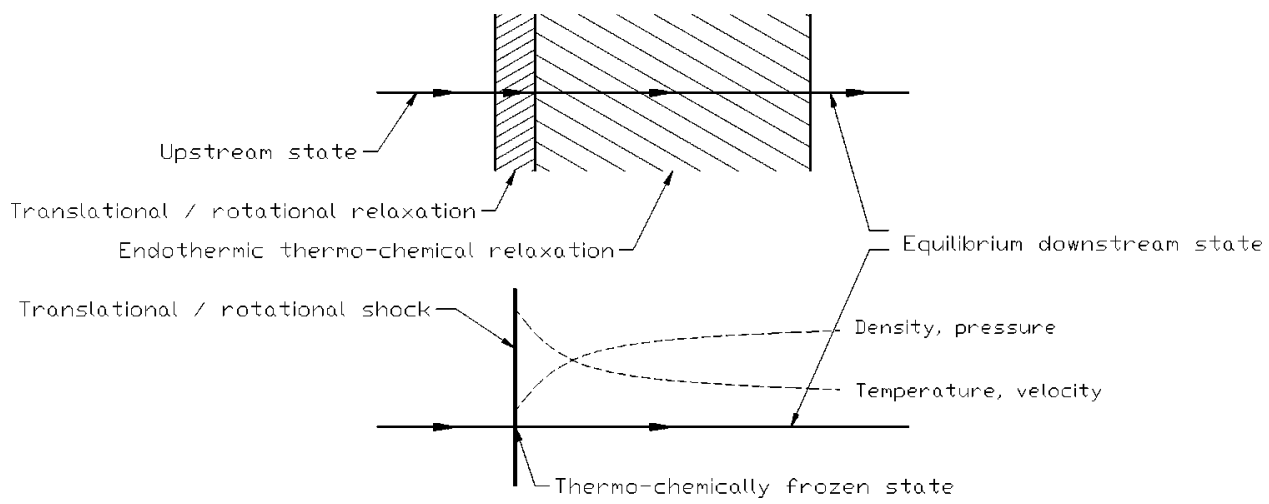

FIG. 1. Model shock structure. The region of translational/rotational nonequilibrium is treated as a gas dynamical discontinuity and only thermochemical reactions on length scales comparable to the fluid scales are modeled. Typical profiles are shown for the density, pressure, temperature and velocity (see also Fig. 5).

We use Lighthill's model ${ }^{2}$ for the thermodynamics of the system (1),

$$
\begin{aligned}
& p=\frac{k}{2 m}(1+\alpha) \rho T, \\
& h=\frac{k}{2 m}\left[(4+\alpha) T+\alpha \theta_{d}\right],
\end{aligned}
$$

where $k$ is Boltzmann's constant, $m$ is the mass of one atom of the gas, $T$ is the temperature, $\theta_{d}$ is a temperature characterizing the dissociation energy and $\alpha$ is the dissociated mass fraction determined from the number densities, $\alpha=n_{N} /\left(n_{N}\right.$ $+2 n_{N_{2}}$ ). Using the caloric equation of state (9) and the dimensionless continuity equation (5), the dimensionless energy conservation equation becomes

$$
\left(4+\alpha_{2}\right) \frac{\hat{T}}{\Theta_{1}}+\alpha_{2}+\frac{K_{1}}{\hat{\rho}^{2}}=H_{0_{1}},
$$

where the dimensionless parameters are defined as

$$
\begin{aligned}
& \Theta_{1}=\frac{\theta_{d}}{T_{1}}, \\
& K_{1}=\frac{m u_{1}^{2}}{k \theta_{d}}, \\
& H_{0_{1}}=\frac{2 m h_{0_{1}}}{k \theta_{d}} .
\end{aligned}
$$

The conserved stagnation enthalpy, $H_{0_{1}}$, is normalized with respect to the dissociation energy of the gas and $K_{1}$ is the normalized specific kinetic energy of the upstream flow.

Importantly, for a nonequilibrium binary mixture of thermally perfect components, three parameters are sufficient to define the state of the upstream gas. Here we specify $P_{1}$, $H_{0_{1}}$ and $\alpha_{1}$. The remaining parameters, $\Theta_{1}$ and $K_{1}$, are then given by the following identities, obtained from $h_{0}$ written in terms of the conditions upstream of the discontinuity:

$$
\begin{gathered}
\Theta_{1}=\frac{4+\alpha_{1}}{H_{0_{1}}-K_{1}-\alpha_{1}}, \\
K_{1}=\frac{H_{0_{1}}-\alpha_{1}}{1+2 P_{1} \frac{4+\alpha_{1}}{1+\alpha_{1}}} .
\end{gathered}
$$

From Eq. (8) the dimensionless form of the thermal equation of state becomes

$$
\hat{T}=\frac{\hat{p}\left(1+\alpha_{1}\right)}{\hat{\rho}\left(1+\alpha_{2}\right)} .
$$

Eliminating $\hat{T}$ and $\Theta_{1}$ from Eq. (10) with the pressure given by (6) leads to the following quadratic equation for the density:

$$
\left(H_{0_{1}}-\alpha_{2}\right) \hat{\rho}^{2}-2 K_{1}\left(1+P_{1}\right) \frac{4+\alpha_{2}}{1+\alpha_{2}} \hat{\rho}+K_{1} \frac{7+\alpha_{2}}{1+\alpha_{2}}=0 .
$$

The solutions are

$$
\begin{aligned}
\hat{\rho}= & \frac{K_{1}}{\left(1+\alpha_{2}\right)\left(H_{0_{1}}-\alpha_{2}\right)} \\
& \times\left[\left(1+P_{1}\right)\left(4+\alpha_{2}\right) \pm\left[\frac{\left(1+\alpha_{2}\right)\left(7+\alpha_{2}\right)\left(\alpha_{2}-H_{0_{1}}\right)}{K_{1}}\right.\right. \\
& \left.\left.+\left(1+P_{1}\right)^{2}\left(4+\alpha_{2}\right)^{2}\right]^{1 / 2}\right] .
\end{aligned}
$$

One solution describes the variation of density throughout the relaxation region downstream of the translational shock. The remaining solution describes relaxation of the possibly nonequilibrium upstream state without any discontinuity. Both solutions are parametrized in terms of the dissociation mass fraction, $\alpha_{2}$, whose rate of reaction remains indeterminate. The solutions are illustrated for typical values of the parameters in Figs. 2 and 3. The result, (19), applies generally in the case $\alpha_{1} \neq 0$ that is required for the solution of shock interaction problems. The dependence of the solution on $\alpha_{1}$ is confined entirely to the dependence of $K_{1}$ on $\alpha_{1}$ according to Eq. (15).

It is worth noting the relationship between the model discussed here and the Zeldovich-von Neumann-Döring (ZND) model for exothermic detonation waves. In the simplest form, the ZND model treats the reactants and products as perfect gases with heat release being modeled by an Arrhenius reaction rate expression. This leads to an induction zone of low reaction rate downstream of the gas dynamical shock and the reaction subsequently accelerates rapidly until progress is limited by depletion of the reactants. 


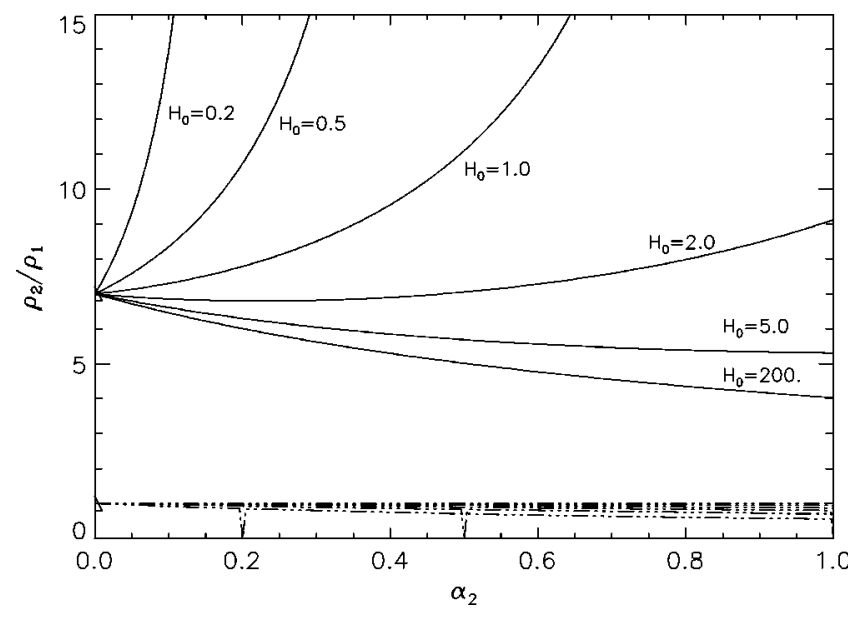

FIG. 2. Strong shock solutions, $P \rightarrow 0$, of the conservation equations downstream of a normal shock wave in an ideal dissociating gas. The upstream conditions are taken as $P_{1}=0, \alpha_{1}=0$ and solutions are plotted for differing values of $H_{0_{1}}$. The two chemically frozen solutions, $\alpha_{1}=\alpha_{2}=0$, are marked on the vertical axis $(\triangle)$. The solid curves represent the relaxation of the density downstream of the translational discontinuity. For moderate values of $H_{0}$ the density increases monotonically as the flow relaxes towards chemical equilibrium. For $H_{0_{1}}>(7 / 6)^{2}$ the density initially decreases. As $H_{0_{1}} \rightarrow \infty$ the solution asymptotes to $\hat{\rho}=4$ which is the strong shock limit for a monatomic gas. At these enthalpies the model is inaccurate since it does not model the electronic excitation that would occur. Each of the solutions exhibits a singularity at $H_{0_{1}}-\alpha_{2}=0$. Beyond this singularity the specific kinetic energy downstream of the relaxation zone becomes negative as may be observed from Eq. (15). The second set of solutions shown by the $-\cdots-$ curves represent the relaxation of the upstream state without a translational discontinuity. This effect becomes larger at high enthalpies as the upstream state $\alpha_{1}=0$ deviates farther from equilibrium.

In the following analysis we concentrate on cases where $\alpha_{1} \approx 0$ since these cases are important for many problems in hypervelocity flow. The limit $\alpha_{1} \rightarrow 1$ is considered since it allows a comparison with known results for monatomic gases. Although it is not our primary motivation, the case $\alpha_{1} \rightarrow 1$ also represents an exothermic recombination shock. There is some concordance between the results obtained in the limit $\alpha_{1} \rightarrow 1$ and those that might be obtained if the current analysis were replicated using the ZND detonation model noted above.

\section{A. Frozen shock solutions}

Consider the frozen solutions that apply directly downstream of the translational discontinuity. Here $\alpha_{2}=\alpha_{1}$ and the solutions of the quadratic expression (17) are

$$
\hat{\rho}_{f}=\left\{\begin{array}{l}
1, \\
\frac{7+\alpha_{1}}{\left(1+\alpha_{1}\right)+2 P_{1}\left(4+\alpha_{1}\right)} .
\end{array}\right.
$$

In the strong shock limit, $P_{1} \rightarrow 0$, this simplifies to

$$
\hat{\rho}_{f}=\left\{\begin{array}{l} 
\begin{cases}1, & \alpha_{1}=0, \\
7, & \end{cases} \\
\begin{cases}1, & \alpha_{1}=1 . \\
4, & \end{cases}
\end{array}\right.
$$

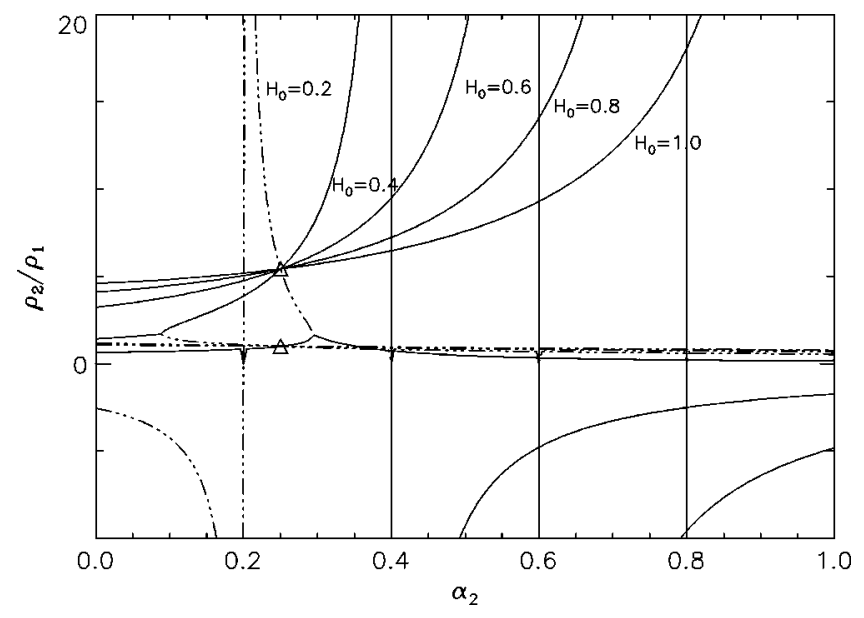

FIG. 3. Relaxation of a nonequilibrium upstream state behind a normal shock wave in an ideal dissociating gas. The upstream state is taken as $P_{1}$ $=0.01, \alpha_{1}=0.25$, and $H_{0_{1}}=0.2 \rightarrow 1.0$. We see that the frozen solutions $(\triangle)$ are displaced from the vertical axis as a result of the upstream dissociation level. The interpretation of the two sets of solutions that are indicated by the solid and dot-dash curves is qualitatively similar to Fig. 2 for the cases $H_{0_{1}}=0.6,0.8,1.0$. The case $H_{0_{1}}=0.4$ corresponds to a recombination shock where the exothermic relaxation process causes the density to decrease downstream of the shock. The case $H_{0_{1}}=0.2$ is physically unrealistic since for values of $H_{0_{1}}<\alpha_{1}$ the specific kinetic energy of the flow is negative.

These solutions are consistent with the limiting cases of a partially vibrationally excited diatomic gas and a monatomic gas, respectively. Note also the value $P_{\max }=3 /\left(4+\alpha_{1}\right)$ which produces $\hat{\rho}_{f}=1$ and corresponds to sonic flow upstream of the shock wave.

\section{B. Equilibrium shock solution}

Equation (17) may be combined with an equilibrium expression to obtain the asymptotic state far downstream from the translational shock. This determines the endpoints for the trajectories shown in Figs. 2 and 3. For the equilibrium ideal dissociating gas (Lighthill ${ }^{2}$,

$$
\frac{\alpha_{2_{\mathrm{eq}}}^{2}}{1-\alpha_{2_{\mathrm{eq}}}}=\frac{\rho_{d}}{\rho_{\mathrm{eq}}} e^{-\theta_{d} / T} .
$$

Writing this in dimensionless form, using the identity (14) and expressing the temperature ratio in terms of the density ratio leads to a second transcendental equation relating $\alpha_{2}$ and $\hat{\rho}$ :

$$
\frac{\alpha_{2_{\mathrm{eq}}}^{2}}{1-\alpha_{2_{\mathrm{eq}}}}=\frac{\hat{\rho}_{d_{1}}}{\hat{\rho}_{\mathrm{eq}}} \exp \left[\frac{\left(1+\alpha_{2_{\mathrm{eq}}}\right) \hat{\rho}_{\mathrm{eq}}^{2}}{2 K_{1}\left(1-\hat{\rho}_{\mathrm{eq}}\left(1+P_{1}\right)\right)}\right] .
$$

An additional dimensionless equilibrium constant is introduced,

$$
\hat{\rho}_{d_{1}}=\rho_{d} / \rho_{1} .
$$

Numerically solving Eq. (17) with $\alpha_{2}=\alpha_{2_{\text {eq }}}$ given by (21) gives the equilibrium shock solution for specified upstream conditions $P_{1}, H_{0_{1}}$ and $\alpha_{1}$. This solution is represented graphically in Fig. 4 by the intersection of the two curves in the $\hat{\rho}$ - $\alpha$ plane defined by Eqs. (17) and (21). When solving 


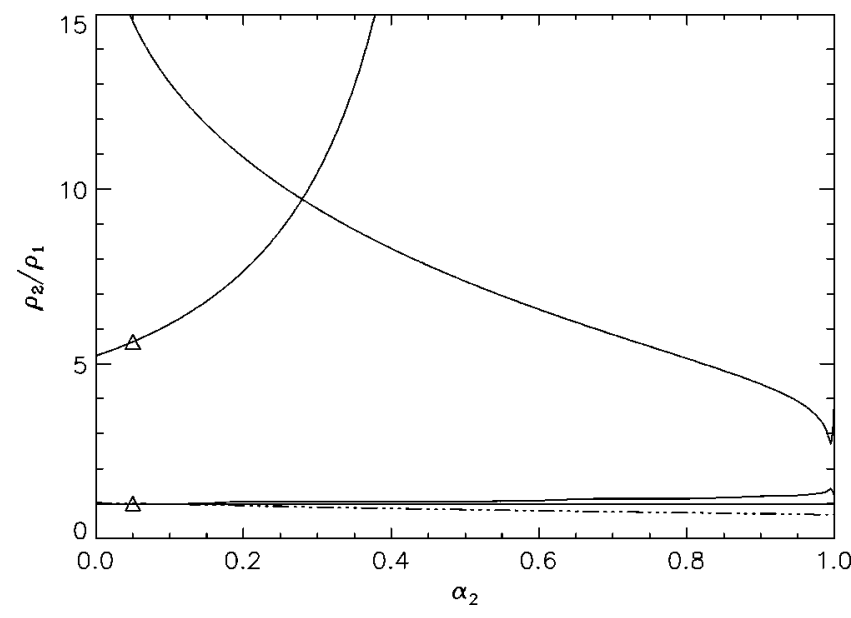

FIG. 4. Equilibrium shock solution in the $\hat{\rho}-\alpha_{2}$ plane. The free-stream conditions are $P_{1}=0.025, H_{0_{1}}=0.55, \hat{\rho}_{d_{1}}=1 \times 10^{7}, \alpha_{1}=0.05$.

these equations numerically the solution is bracketed in the intervals $\hat{\rho}_{2_{\mathrm{eq}}} \in\left[\hat{\rho}_{f}, \infty\right)$ and $\alpha_{2_{\mathrm{eq}}} \in\left[\alpha_{1}, 1\right]$. This enables the development of a robust solver with known convergence properties in the troublesome weak shock limit. For the routine construction of $p$ - $\delta$ loci this is a significant concern.

\section{Nonequilibrium solution}

The rate at which the dissociation reaction proceeds from the frozen state to the equilibrium state for the IDG model is given by Freeman, ${ }^{3}$

$$
\frac{d \alpha_{2}}{d x}=\frac{d \alpha_{2}}{d t} \frac{d t}{d x}=\frac{C \rho T^{\eta}}{u}\left[\left(1-\alpha_{2}\right) e^{-\theta_{d} / T}-\frac{\rho}{\rho_{d}} \alpha_{2}^{2}\right],
$$

where $C$ is the reaction rate constant and $\eta$ represents the pre-exponential temperature dependence of the reaction rate (see Table I). Defining the characteristic reaction length,

$$
\ell_{d_{1}}=\frac{u_{1}}{C \rho_{1} \theta_{d}^{\eta}},
$$

and applying the previous dimensionless notation this becomes

$$
\frac{d \alpha_{2}}{d \hat{x}}=\ell_{d_{1}} \frac{d \alpha_{2}}{d x}=\hat{\rho}^{2} \hat{T}^{\eta} \Theta_{1}^{-\eta}\left[\left(1-\alpha_{2}\right) e^{-\Theta_{1} / \hat{T}}-\frac{\hat{\rho}}{\hat{\rho}_{d_{1}}} \alpha_{2}^{2}\right] .
$$

Eliminating the temperature dependence using Eqs. (16), (6), and (14) we obtain,

$$
\frac{\Theta_{1}}{\hat{T}}=\frac{\hat{\rho}^{2}\left(1+\alpha_{2}\right)}{2 K_{1}\left(\hat{\rho}\left(P_{1}+1\right)-1\right)} .
$$

TABLE I. Constants for IDG model.

$$
\begin{gathered}
\theta_{d}=113200 \mathrm{~K} \\
\rho_{d}=130000 \mathrm{~kg} \mathrm{~m}^{-3} \\
m=14.0 \times 10^{-3} / 6.023 \times 10^{23} \mathrm{~kg} \\
C=2.7 \times 10^{21} \mathrm{~m}^{3} \mathrm{~kg}^{-1} \mathrm{~s}^{-1} \mathrm{~K}^{2.5} \\
\eta=-2.5
\end{gathered}
$$
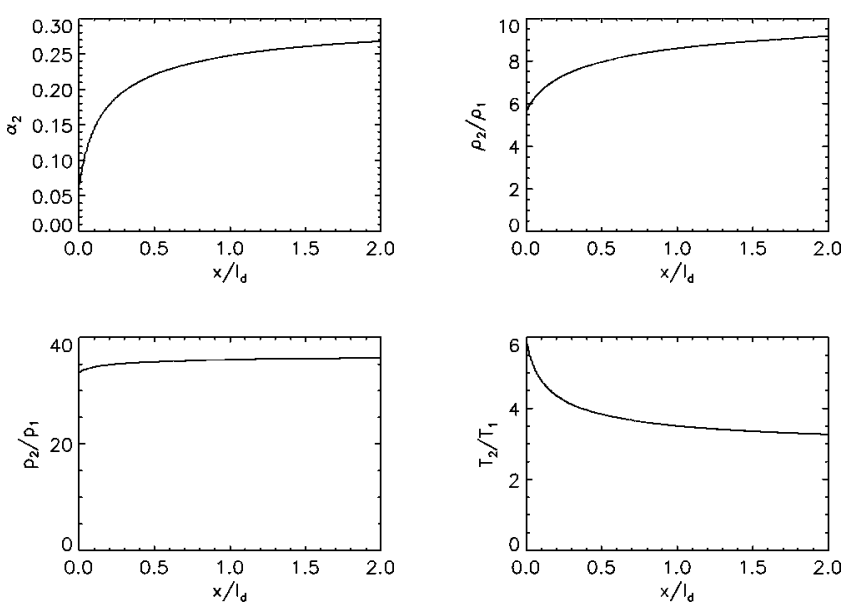

FIG. 5. Nonequilibrium solution for the relaxation downstream of a translational discontinuity in an ideal dissociating gas. The free stream conditions are $P_{1}=0.0254, H_{0_{1}}=0.56, \hat{\rho}_{d_{1}}=8.3 \times 10^{6}, \alpha_{1}=0.051, \eta=-2.5$.

Equations (25) and (26) define a third relation between $\alpha_{2}$ and $\hat{\rho}$ that, additionally, involves the distance $\hat{x}=x / \ell_{d_{1}}$ from the translational discontinuity. While (17) defines the relaxation in $\alpha_{2}-\hat{\rho}$ space between the frozen state and the equilibrium solution, integration of the ordinary differential equation relates $\alpha_{2}$ and $\hat{\rho}$ to $\hat{x}$. The resulting relaxation profiles are shown in Fig. 5.

\section{Reaction rate parameter}

The reaction rate immediately downstream of the translational discontinuity, when normalized with respect to the rate of flow over a blunt body, represents a useful measure of the departure of the flow field from equilibrium. This leads to a definition of the dissociation rate parameter,

$$
\Delta=\left.\frac{d \alpha_{2}}{d t}\right|_{f} \frac{D}{u_{1}}=\left.\frac{d \alpha_{2}}{d x}\right|_{f} u_{2} \frac{D}{u_{1}}=\left.\frac{d \alpha_{2}}{d \hat{x}}\right|_{f} \frac{D}{\hat{\rho}_{f} \ell_{d_{1}}},
$$

where $D$ is the diameter of the body, $\ell_{d_{1}}$ is the characteristic reaction length and the subscript, $f$, refers to the frozen state immediately downstream of the translational discontinuity. The initial dissociation rate, $d \alpha_{2} /\left.d \hat{x}\right|_{f}$, is determined by the exponential term of Eq. (25) for the frozen state downstream of the shock, where $\alpha_{2}=\alpha_{1}$. Equation (19) gives the frozen solution for the density and the temperature dependence follows from (26):

$$
\Delta=\frac{D}{\ell_{d_{1}}} \hat{\rho}_{f}\left(\frac{\Theta_{1}}{\hat{T}_{f}}\right)^{-\eta}\left(1-\alpha_{1}\right) e^{-\Theta_{1} / \hat{T}_{f}}
$$

This parameter is linearly dependent on the ratio of the body size, $D$, to the characteristic reaction length, $\ell_{d_{1}}$. The remaining terms are most strongly dependent on the stagnation enthalpy, $H_{0_{1}}$. This may be demonstrated in the hypersonic limit, $P_{1} \rightarrow 0$, with $\alpha_{1}=0$. The frozen density ratio reduces to $\hat{\rho}_{f} \rightarrow 7 /\left(1+8 P_{1}\right) \rightarrow 7$ and hence $\Theta_{1} / \hat{T} \rightarrow 7^{2} / 12 K_{1}$ $\rightarrow 7^{2} / 12 H_{0_{1}}$. In this limit we obtain 


$$
\Delta \simeq 7 \frac{D}{\ell_{d_{1}}}\left(\frac{49}{12 H_{0_{1}}}\right)^{-\eta} e^{-49 / 12 H_{0_{1}}} .
$$

This is a good approximation for a wide range of the parameters since the general form, given by Eqs. (27) and (26), depends only weakly on $P_{1}$ and $\alpha_{1}$. The rationale for binary scaling (see Hall, Eschenroeder, and Marrone ${ }^{4}$ ) that is used to achieve dimensional similitude in hypervelocity shock tunnels is apparent from the form of Eq. (28) since this gives similarity in terms of $H_{0_{1}}$ and $\Delta$.

Typical values of the physical constants for the IDG model of nitrogen are given in Table I.

\section{EXTENSION TO OBLIQUE SHOCK WAVES}

\section{A. Normal components of the free stream parameters}

The extension to oblique waves is obtained by the conventional technique of applying the previous solutions to the component of velocity normal to the shock front. If $\beta$ is the angle of the oblique shock wave, then from the definitions of $P_{1}$ and $K_{1}$ we obtain the normal components,

$$
\begin{aligned}
& P_{1_{N}}=\frac{p_{1}}{\rho_{1} u_{1}^{2} \sin ^{2} \beta}=\frac{P_{1}}{\sin ^{2} \beta}, \\
& K_{1_{N}}=\frac{m u_{1}^{2} \sin ^{2} \beta}{k \theta_{d}}=K_{1} \sin ^{2} \beta .
\end{aligned}
$$

Here and in the material that follows, the subscripts $N$ and $T$ refer to the components normal and tangential to the shock front, respectively. The identity (15) then gives the normal component of the enthalpy,

$$
H_{0_{1_{N}}}=K_{1_{N}}\left(1+2 P_{1_{N}} \frac{4+\alpha_{1}}{1+\alpha_{1}}\right)+\alpha_{1} .
$$

Given $H_{0_{1_{N}}}, P_{1_{N}}$, and $\alpha_{1}$ the variation of density downstream of the oblique shock is then determined by Eq. (17). Solving simultaneously with Eq. (21) gives the equilibrium solution and integrating the ODE (25) determines the intermediate states. Once the density is known, the momentum equation, (6), gives the pressure variation along the streamline. The variation of the flow deflection angle, $\delta$, is determined from continuity and the conservation of velocity tangential to the shock to be

$$
\tan (\beta-\delta)=\frac{\tan \beta}{\hat{\rho}} .
$$

This result carries over directly from the perfect gas case and here it applies throughout the relaxation zone downstream of a straight oblique shock wave.

The parametric dependence of some arbitrary dimensionless quantity, $\hat{\phi}$, downstream of an oblique shock wave may then be summarized as

$$
\hat{\phi}=\hat{\phi}\left[P_{1}, H_{0_{1}}, \alpha_{1}, \hat{\rho}_{d_{1}}, x / \ell_{d_{1}}, \eta, \beta\right],
$$

where $P_{1}$ behaves as $P_{1} \sim 1 / M_{1}^{2}, H_{0_{1}}$ is the dimensionless stagnation enthalpy, $\alpha_{1}$ is the upstream dissociation level, $\hat{\rho}_{d_{1}}$ is the equilibrium constant, $x / \ell_{d_{1}}$ is the dimensionless distance normal to the shock front, $\eta$ is the temperature exponent of the reaction rate and $\beta$ is the shock wave angle. The behavior for typical values of the parameters is shown in Fig. 6.

\section{B. Variation of dimensionless parameters across an oblique shock wave}

To facilitate the computation of multiple shock jumps in the solution of interaction problems we must find a convenient means of determining the downstream dimensionless parameters, that become the upstream parameters for subsequent shock waves. Convenient forms are required for $P_{2}$, $H_{0_{2}}, \hat{\rho}_{d_{2}} / \hat{\rho}_{d_{1}}$ and $\ell_{d_{2}} / \ell_{d_{1}}$. Since stagnation enthalpy is conserved,

$$
H_{0_{2}}=H_{0_{1}} \text {. }
$$

In order to determine $P_{2}$ observe that $u_{2}^{2}=u_{2}{ }^{2}+u_{2_{N}}{ }^{2}$ and so $K_{2}=K_{2_{T}}+K_{2_{N}}$. Further, since $u_{1_{T}}=u_{2_{T}}$ and $\hat{\rho} \hat{u}_{N}=1$, it follows that

$$
K_{2}=K_{1}\left(\cos ^{2} \beta+\frac{\sin ^{2} \beta}{\hat{\rho}^{2}}\right) .
$$

Identity (15) then yields $P_{2}$ and the computation for the downstream shock wave proceeds as before using $P_{2}, H_{0_{2}}$, and $\alpha_{2}$ as the new upstream condition. Downstream of the shock we must adjust the equilibrium constant according to

$$
\hat{\rho}_{d_{2}}=\frac{\hat{\rho}_{d_{1}}}{\hat{\rho}} .
$$

Again, these relations apply throughout the relaxation zone. The relations may be applied recursively for problems involving series of shock waves.

The normalization of the reaction rates downstream of a sequence of shock waves must be adjusted to a common basis for comparison and this is chosen to be the characteristic reaction length for the free stream, $\ell_{d_{\infty}}$. At this point we adjust our notation slightly for multiple wave interaction problems. The subscript, $\infty$, refers to the free stream, and a numerical subscript identifies a shock wave and refers to the downstream state. For the $i$ th wave in a series of shocks we then have

$$
\frac{d \alpha_{i}}{d \hat{x}_{\infty}}=\frac{d \alpha_{i}}{d \hat{x}_{i_{N}}} \frac{d \hat{x}_{i_{N}}}{d x_{i_{N}}} \frac{d x_{\infty}}{d \hat{x}_{\infty}} \frac{d x_{i_{N}}}{d x_{\infty}} .
$$

The first term on the right-hand side, $d \alpha_{i} / d \hat{x}_{i_{N}}$, is given by Eq. (25) using the normal components of the parameters for the $i$ th shock. The remaining terms that adjust the normalization are

$$
\begin{aligned}
& \frac{d \hat{x}_{i_{N}}}{d x_{i_{N}}}=\frac{1}{\ell_{d_{i_{-1} N}}}=\frac{\rho_{i_{-1}} \theta_{d}^{\eta} C}{u_{i_{-1} N}}, \\
& \frac{d x_{\infty}}{d \hat{x}_{\infty}}=\ell_{d_{\infty}}=\frac{u_{\infty}}{\rho_{\infty} \theta_{d}^{\eta} C},
\end{aligned}
$$



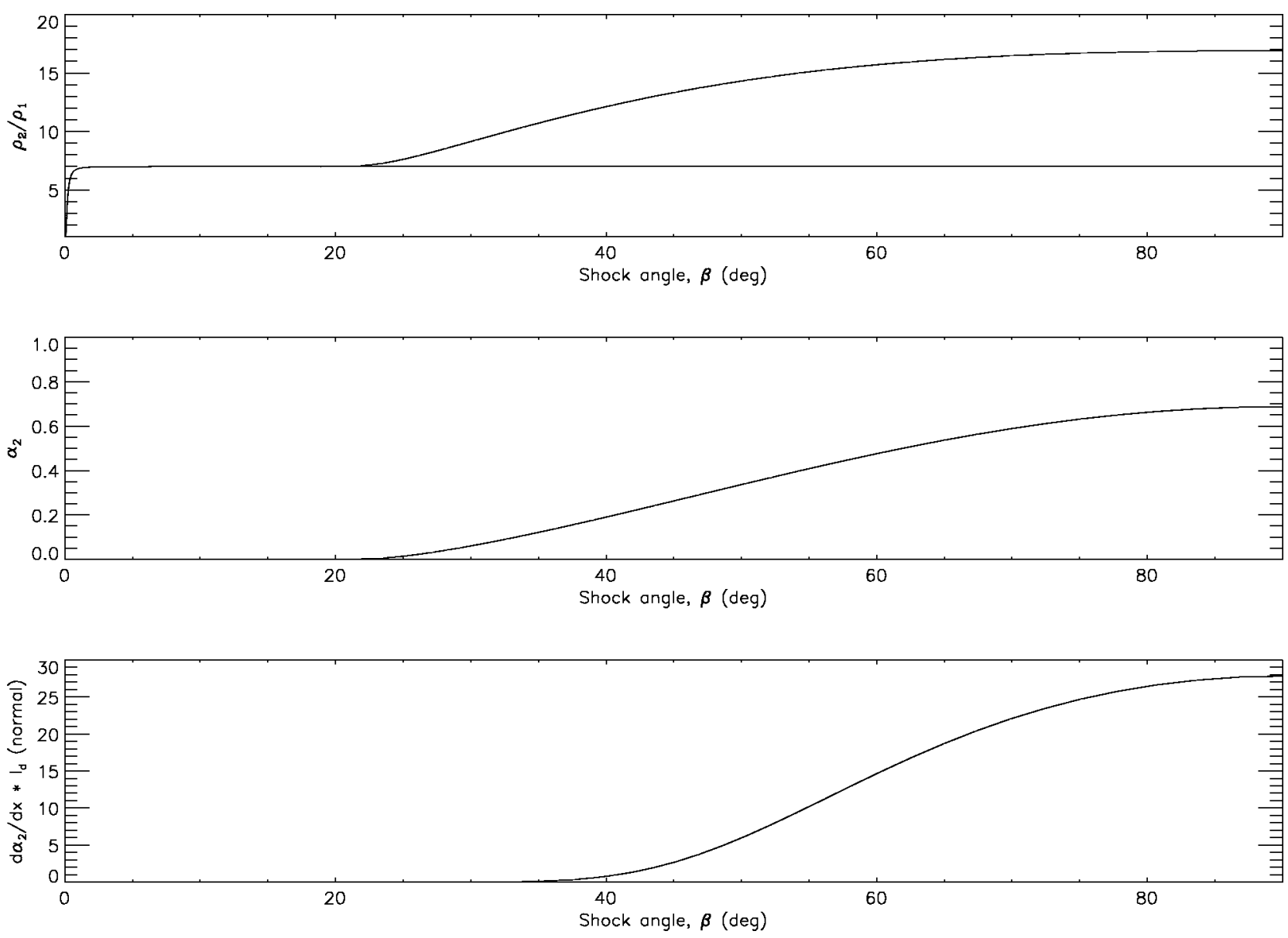

FIG. 6. Frozen and equilibrium density ratio, equilibrium dissociation level, and initial dissociation rate as functions of shock angle for an oblique wave in an ideal dissociating gas. The free stream conditions approach the hypersonic limit $P_{1}=0\left(P_{1}=1 \times 10^{-6}, H_{0_{1}}=1.0, \alpha_{1}=0, \hat{\rho}_{d_{1}}=1 \times 10^{8}\right)$. Since we illustrate only the frozen and equilibrium limits, the dependence on $x / \ell_{d_{1}}$ and $\eta$ disappears. The dual mechanisms of compressibility and dissociation cause nonmonotonic behavior of the shock density ratio with an increasing shock angle. Even for very weak shock waves the Mach number normal to the shock wave is sufficient to cause the density to approach the strong shock limit for frozen chemistry. Only at larger shock angles do the frozen and equilibrium solutions deviate as the exponential dependence of the reaction rate and the equilibrium dissociation fraction on the normal component of the enthalpy becomes significant. The density rises in direct relation to the downstream dissociation level. Note, however, that for waves of moderate strength that produce low dissociation levels, the reaction rates remain small.

$$
\frac{d x_{i_{N}}}{d x_{\infty}}=\sin \left(\beta_{i}+\sum_{j=1}^{i_{-j}=1} \delta_{i_{-j}}\right) .
$$

The notation, $i_{-j}$, refers to the sequence of upstream shocks involved in the normalization (e.g., for a shock, $i=6$ with waves $i=5, i=3$ and $i=1$ upstream of it; $i_{-j} \in[5,3,1]$ with $i_{-1}=5, i_{-2}=3$, etc.). Substituting these relations into Eq. (37) and using the definition of the dimensionless specific kinetic energy, $K$, we obtain the consistently normalized reaction rate

$$
\frac{d \alpha_{i}}{d \hat{x}_{\infty}}=\frac{d \alpha_{i}}{d \hat{x}_{i_{N}}} \sqrt{\frac{K_{\infty}}{K_{i_{-1} N}}} \prod_{j=0}^{i_{-j}=1} \hat{\rho}_{i_{-j}} \sin \left(\beta_{i}+\sum_{j=1}^{i_{-j}=1} \delta_{i_{-j}}\right) .
$$

\section{SHOCK WAVE INTERACTIONS}

\section{A. Pressure-flow deflection angle $(\boldsymbol{p}-\delta)$ plane}

The flows in smooth regions of shock interaction flow fields and across shock discontinuities are, in principle, understood. The predominant concern is then points where sur- faces of discontinuity intersect and here local analysis about intersection points in the pressure-flow deflection angle ( $p$ $\delta$ ) plane provides a useful tool. These techniques were developed in the study of Mach reflection problems and reviews are given by Courant and Friedrichs ${ }^{5}$ and more recently by Hornung. ${ }^{6}$ In this method the solution is represented graphically by the intersection in the $p$ - $\delta$ plane of the loci of possible downstream states for the interacting waves.

The influence of real gas effects on this construction is illustrated for a single oblique shock wave in Fig. 7. The methodology for determination of the generic states, (1) and (2), across a shock wave was described in Sec. III. Here it is described functionally by the relationship

$$
\left(\hat{\rho}_{2}, \hat{p}_{2}, \delta_{2}, P_{2}, H_{0_{2}}, \alpha_{2}\right)=\mathcal{J}\left(P_{1}, H_{0_{1}}, \alpha_{1}, \hat{\rho}_{d}, \beta_{1}\right)
$$

The shock jump conditions, $\mathcal{J}$, are obtained from Eq. (17) combined with Eq. (19) in the frozen limit or with Eq. (21) in the equilibrium limit. Normal components of the parameters are used as determined in Sec. III. The resulting solu- 


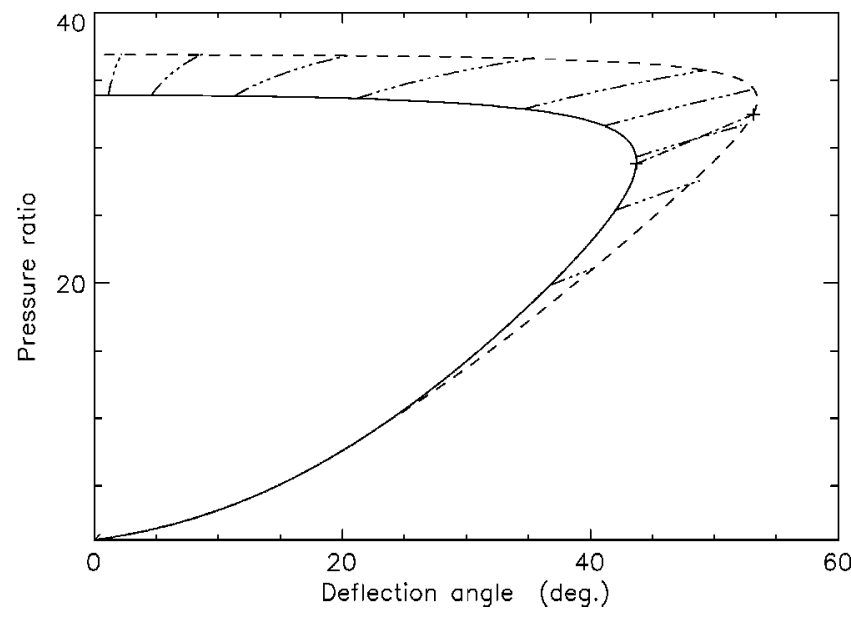

FIG. 7. The $p$ - $\delta$ plane representation of an oblique shock wave for an ideal dissociating gas. The solid curve is the frozen locus. The dashed curve is the equilibrium locus. - $\cdots-$ are streamlines for shock angles $\beta=40^{\circ}, 50^{\circ}$, $60^{\circ}, 68.5^{\circ}, 75^{\circ}, 80^{\circ}, 85^{\circ}, 87.5^{\circ}, 89^{\circ}$, and $89.75^{\circ}$, respectively. - - is the mapping of the frozen sonic line connecting the frozen and equilibrium limits $(+)$. The free stream conditions are $P_{1}=0.025, H_{0_{1}}=0.55$, $\hat{\rho}_{d_{1}}=1 \times 10^{7}, \alpha_{1}=0.05$.

tions apply directly at the intersection point or far from the shock fronts, respectively. Near and far are interpreted relative to the characteristic reaction length, $\ell_{d_{1}}$.

Real gas effects cause the frozen and equilibrium loci to be separated by amounts that increase monotonically with $H_{0_{1}}$ (see Fig. 7). For endothermic reactions both the pressure and flow deflection angle increase across the relaxation zone. The well known insensitivity of the pressure to gas dissociation is evident by limited vertical separation of the loci. Nonequilibrium effects are most pronounced in the flow deflection behind strong waves whereby $H_{0_{N}}$ is large. For nearly normal waves the tangential component of velocity is of the same order as the normal velocity and so relaxation produces a large change in the flow deflection angle. This may be observed in the streamlines that are mapped into the $p$ - $\delta$ plane in Fig. 7. Streamlines are defined by Eq. (17) and are parametrized in terms of $\alpha_{2}$; where $\alpha_{2} \in\left[\alpha_{1}, \alpha_{2}\right]$ for each $\beta$. The pressure and flow deflection angle are given by Eqs. (6) and (32) for each value of $\alpha_{2}$. In the weak shock limit the origin of the frozen and equilibrium loci in Fig. 7 do not coincide when $\alpha_{1}$ is nonzero and sufficiently large. This is a consequence of relaxation of a nonequilibrium upstream state (recall the behavior of the second branch of the shock wave solutions shown in Figs. 2 and 3).

\section{B. Mapping of the sonic line}

Consider the differential forms of the thermal equation of state, (8),

$$
d p=p_{\rho} d \rho+p_{T} d T+p_{\alpha} d \alpha,
$$

and of the caloric equation of state for a mixture of thermally perfect components, (9),

$$
d h=h_{T} d T+h_{\alpha} d \alpha .
$$

For an adiabatic, thermodynamically reversible system we have, $d e=p d v$ or equivalently $d h=d p / \rho$, so that

$$
\frac{d p}{\rho}=h_{T} d T+h_{\alpha} d \alpha .
$$

Eliminating $d T$ between Eqs. (40) and (42) we have

$$
\left(h_{T}-\frac{p_{T}}{\rho}\right) d p=h_{T} p_{\rho} d \rho+\left(h_{T} p_{\alpha}-p_{T} h_{\alpha}\right) d \alpha .
$$

In the case of frozen perturbations where $d \alpha=0$, the chemically frozen sound speed, $a_{f}$, then follows directly from Eq. (43) as

$$
a_{f}^{2}=\left.\frac{d p}{d \rho}\right|_{f}=\frac{h_{T} p_{\rho}}{h_{T}-p_{T} / \rho}=\frac{p_{\rho}}{1-\frac{p_{T}}{h_{T} \rho}} .
$$

Forming the thermodynamic derivatives from Eqs. (8) and (9) we have the usual result,

$$
a_{f}^{2}=\frac{k}{2 m} \frac{(1+\alpha)(4+\alpha)}{3} T,
$$

or in the dimensionless notation used here,

$$
M_{f}^{2}=\frac{u^{2}}{a_{f}^{2}}=\frac{3}{(4+\alpha) P} .
$$

Define a function $q(\rho, T, \alpha)$ that expresses the departure of the system from thermochemical equilibrium, as determined by the law of mass action (20),

$$
q(\rho, T, \alpha)=\frac{\alpha^{2}}{1-\alpha}-\frac{\rho_{d}}{\rho} e^{-\theta_{d} / T} .
$$

For a system in equilibrium the differential is identically zero,

$$
d q=q_{\rho} d \rho+q_{T} d T+q_{\alpha} d \alpha=0 .
$$

Eliminating $d T$ between Eqs. (41) and (48) we have

$$
\frac{q_{T}}{\rho} d p=-h_{T} q_{\rho} d \rho+\left(q_{T} h_{\alpha}-h_{T} q_{\alpha}\right) d \alpha .
$$

Eliminating $d \alpha$ between Eqs. (43) and (49), we obtain the sound speed for a system in thermodynamic and chemical equilibrium,

$$
a_{e}^{2}=\left.\frac{d p}{d \rho}\right|_{e}=\frac{h_{T} p_{\rho} \xi_{1}+h_{T} q_{\rho} \xi_{2}}{\left(h_{T}-p_{T} / \rho\right) \xi_{1}-\left(q_{T} / \rho\right) \xi_{2}},
$$

where for convenience we extract the terms

$$
\xi_{1}=q_{T} h_{\alpha}-h_{T} q_{\alpha}
$$

and

$$
\xi_{2}=h_{T} p_{\alpha}-p_{T} h_{\alpha}
$$

Evaluating the thermodynamic derivatives from Eqs. (8), (9), and (47) and after simplification we obtain

$$
M_{e}^{2}=M_{f}^{2} \frac{(1+\alpha)(4+\alpha)\left[3(\alpha-2)+\alpha \Theta^{2}(\alpha-1)\right]}{3\left[-8+\alpha^{3}(1+\Theta)^{2}-\alpha(3+\Theta(\Theta+2))\right]} .
$$


Observing that the dissociation levels behind oblique shock waves up to the transition points remain relatively weak and $\Theta=O(1)$, it is instructive to linearize Eq. (53) in the limit $\alpha \rightarrow 0$,

$$
M_{e}^{2} \approx M_{f}^{2}\left[1+\frac{(\Theta-3)^{2}}{24} \alpha+O\left(\alpha^{2}\right)\right], \quad \alpha \rightarrow 0 .
$$

The equilibrium Mach number is therefore larger than the frozen Mach number (at the equilibrium composition) and indeed the general result (53) is positive definite over the range of interest.

The equilibrium Mach number only has meaning at the equilibrium condition reached downstream of the thermochemical relaxation zone and in the limit of fast reaction rates. In contrast, the frozen Mach number has meaning throughout the continuum of states that occur in the relaxation zone. Equation (46) may be applied for any value $\alpha$ $\in\left[\alpha_{1}, \alpha_{2}\right]$. The relationship $\mu\left(M_{f_{2}}, \hat{\rho}, \alpha_{2}\right)=0$ between the shock angle, $\beta$, and the frozen Mach number, $M_{f_{2}}$, downstream of an oblique shock wave may be determined from Eqs. (15), (35), and (46),

$$
\begin{aligned}
& \mu\left(M_{f_{2}}, \hat{\rho}, \alpha_{2}\right) \\
& =\frac{\left(H_{0_{2}}-\alpha_{2}\right)}{1+\frac{6}{M_{f_{2}}^{2}\left(1+\alpha_{2}\right)}}-K_{1}\left(1-\sin ^{2} \beta+\frac{\sin ^{2} \beta}{\hat{\rho}^{2}}\right)=0 .
\end{aligned}
$$

This result applies throughout the relaxation zone, provided that self consistent values of $\hat{\rho}$ and $\alpha_{2}$ are used according to the shock jump quadratic, (17), using the normal components of the free stream parameters [Eqs. (29), (30), and (31)],

$$
\begin{aligned}
j\left(\hat{\rho}, \alpha_{2}\right)= & \left(H_{0_{1_{N}}}-\alpha_{2}\right) \hat{\rho}^{2}-2 K_{1_{N}}\left(1+P_{1_{N}}\right) \frac{4+\alpha_{2}}{1+\alpha_{2}} \hat{\rho} \\
& +K_{1_{N}} \frac{7+\alpha_{2}}{1+\alpha_{2}}=0 .
\end{aligned}
$$

Setting $M_{f_{2}}=1$, we obtain a system of equations (55) and (56), for $\sin ^{2} \beta$ that describes the sonic line, parametrized in terms of $\alpha_{2}$. Given $\beta$ and $\alpha_{2}$, in Sec. IV A we describe the mapping of each point on the sonic line into the $p$ - $\delta$ plane (see Fig. 7).

Although the sonic line may be simply computed using the foregoing methodology, simple analysis highlights the important phenomena. Consider initially the frozen solution immediately downstream of the translational discontinuity, Eq. (19), and eliminate $K_{1}$ from Eq. (55) via Eq. (15) and set $M_{f_{2}}=1, \alpha_{2}=\alpha_{1}$. The following quadratic equation for $\sin ^{2} \beta_{S_{f}}$ is obtained:

$$
\frac{1+\alpha_{1}}{7+\alpha_{1}}-\frac{1-\sin ^{2} \beta_{S_{f}}+\frac{\sin ^{2} \beta_{S_{f}}\left(1+\alpha_{1}+\frac{2\left(4+\alpha_{1}\right) P_{1}}{\sin ^{2} \beta_{S_{f}}}\right)^{2}}{\left(7+\alpha_{1}\right)^{2}}}{1+\frac{2\left(4+\alpha_{1}\right) P_{1}}{1+\alpha_{1}}}
$$$$
=0 \text {, }
$$

with solutions

$$
\begin{aligned}
\sin ^{2} \beta_{S_{f}} & \\
& =\frac{21+\alpha_{1}\left(3-P_{1}\right)-P_{1}\left(20-\alpha_{1}^{2}\right)}{12\left(4+\alpha_{1}\right)} \\
& \pm \frac{\sqrt{48\left(4+\alpha_{1}\right)^{3} P_{1}^{2}+\left(21+\alpha_{1}\left(3-P_{1}\right)-P_{1}\left(20-\alpha_{1}^{2}\right)\right)^{2}}}{12\left(4+\alpha_{1}\right)}
\end{aligned}
$$

Here $\beta_{S_{f}}$ is the shock angle that produces $M_{f_{2}}=1$ immediately downstream of the translational discontinuity. Only the positive root is physically meaningful. In the strong shock limit, $P_{1} \rightarrow 0$, this simplifies to

$$
\sin ^{2} \beta_{S_{f}}=\left\{\begin{array}{l} 
\begin{cases}7 / 8, & \alpha_{1} \rightarrow 0, \\
0, & \\
\begin{cases}4 / 5, & \alpha_{1} \rightarrow 1 \\
0, & \end{cases} \end{cases}
\end{array}\right.
$$

In order to examine the evolution of the frozen Mach number throughout the relaxation zone, consider the differentials in $M_{f_{2}}-\hat{\rho}-\alpha_{2}$ space of the general forms of $\mu\left(M_{f}, \hat{\rho}, \alpha_{2}\right)$ and $j\left(\hat{\rho}, \alpha_{2}\right)[$ Eqs. (55) and (56)],

$$
\begin{aligned}
& d \mu=\mu_{M_{f_{2}}} d M_{f_{2}}+\mu_{\alpha_{2}} d \alpha_{2}+\mu_{\hat{\rho}} d \hat{\rho}=0, \\
& d j=j \alpha_{2} d \alpha_{2}+j \hat{\rho} d \hat{\rho}=0,
\end{aligned}
$$

eliminating $d \hat{\rho}$ we obtain

$$
\frac{d M_{f_{2}}}{d \alpha_{2}}=\frac{j_{\alpha_{2}} \mu_{\hat{\rho}}-\mu_{\alpha_{2}} j_{\hat{\rho}}}{j_{\hat{\rho}} \mu_{M_{f_{2}}}} \text {. }
$$

Equation (62) describes the evolution of the frozen Mach number downstream of an oblique shock wave of fixed angle, $\beta$. The solution is parametrized in terms of $\alpha_{2}$, whose rate of reaction again remains indeterminate. We will not discuss the form of Eq. (62) for general free stream conditions since the solution for the entire sonic line is easily obtained by numerical means. It is instructive, however, to consider the hypersonic limit $P_{1} \rightarrow 0$ with $\alpha_{1}=\alpha_{2}$. Forming the derivatives in Eq. (62), taking the hypersonic limit, and evaluating the expression at the sonic shock angle, $\beta_{S_{f}}$, given by Eq. (59) we obtain 


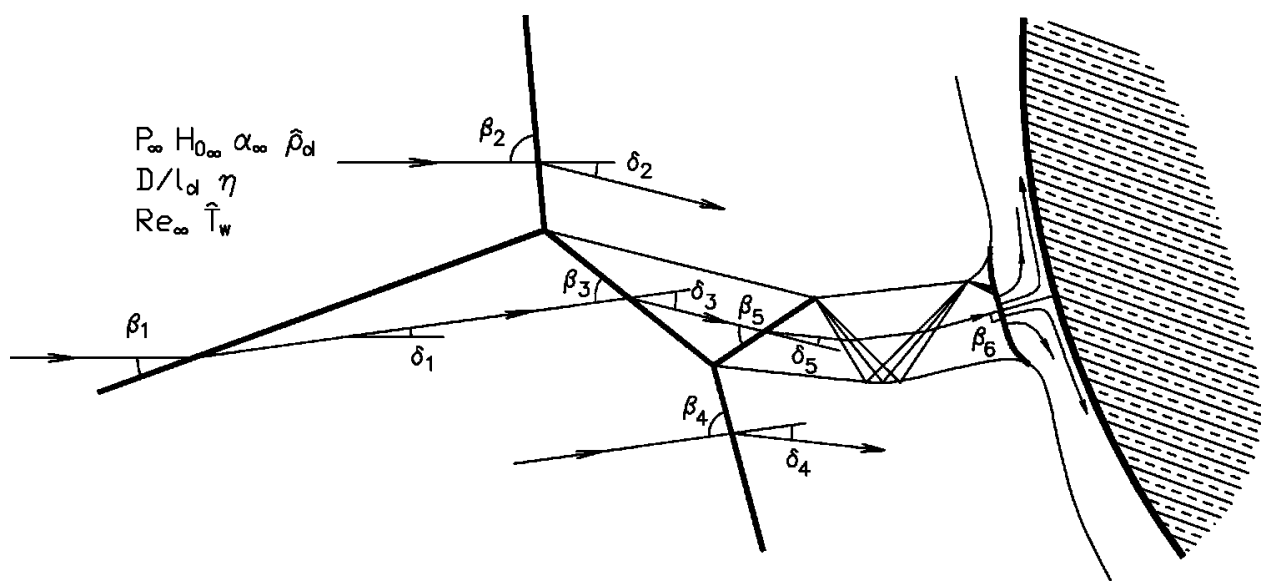

FIG. 8. Schematic of flow field and shock wave notation for the Edney type IV interaction in the vicinity of the impingement point.

$$
\frac{d M_{f_{2}}}{d \alpha_{2}}= \begin{cases}\frac{-3}{8}+\frac{7}{18 H_{0_{1}}}, & \alpha_{1} \rightarrow 0 \\ \frac{67-27 H_{0_{1}}}{180\left(H_{0_{1}}-1\right)}, & \alpha_{1} \rightarrow 1\end{cases}
$$

In the case of a dissociation shock, $\alpha_{1} \rightarrow 0$, we find that $d M_{f_{2}} / d \alpha_{2}>0$ for realistic values of $H_{0_{1}}<28 / 27$. The frozen Mach number therefore increases downstream of the translational discontinuity at the frozen sonic point. This may be interpreted as the case of the insipient formation of a subsonic region of finite extent within the relaxation zone. For shock angles slightly larger than $\beta_{S_{f}}$, we observe in Fig. 7 that the streamline intersects the sonic line. The initially subsonic flow relaxes to a supersonic state as the temperature falls behind the shock front. The resulting formation of a subsonic region of finite extent leads to new phenomena not observed in low enthalpy flows. Scale independent shock wave flow fields, in the limit of fast reaction rates, revert to dependence on the equilibrium sonic point as shown in Fig. 7. These facts were exploited by Hornung and $\mathrm{Smith}^{7}$ to explain the shock detachment process in a relaxing gas and by Hornung, Oertel, and Sandeman ${ }^{9}$ to elucidate the communication of length scales in steady and unsteady relaxing flows.

In the case of a recombination shock with $\alpha_{2}<\alpha_{1}$ and $\alpha_{1} \rightarrow 1$, the solution, (63), is singular at $H_{0_{1}}=1$. For $H_{0_{1}}$ $<1$ we see that $d M_{f} / d \alpha_{2}<0$. Again, this may be interpreted as the point of insipient formation of a subsonic region of finite extent downstream of an exothermic recombination shock (note that here $d \alpha_{2}<0$ along a streamline). For $H_{0_{1}}$ $>1$ we see that $d M_{f} / d \alpha_{2}>0$ and this represents the insipient formation of a supersonic region of finite extent. Although this result is obtained for a recombination shock we have previously noted the relationship to the study of detonation phenomena. Recent experiments by Kaneshige and Shepherd ${ }^{8}$ have studied the role of chemical and fluid mechanical scales on the existence and structure of oblique detonation waves. The data indicate that an oblique detonation front is stabilized by a blunt projectile traveling faster than the Chapman-Jouget velocity provided that the chemical time scales are short compared with the fluid mechanical time scales. Spherical bodies were considered, with the diameter being the only geometric parameter. If one were able to conduct detonation experiments with conical projectiles of varying vertex angle (cf. Hornung and Smith, ${ }^{7}$ who studied endothermic flows) further insight might be gained into the interaction of length scales via the sonic shock structure that occurs when an oblique detonation stabilizes at the tip of a projectile.

\section{Shock wave interaction solutions}

The $\lambda$-pattern of shock waves that is observed to form at the end of a Mach stem is well known. The flow field that arises where a weak shock wave impinges on the leading edge of a blunt body (Edney ${ }^{10,11}$ ) is locally equivalent to the Mach reflection problem. The idealized flow field and the shock wave notation for the important Edney type IV interaction is shown in Fig. 8. In both cases the relevant system of equations is

$$
\begin{aligned}
& \left(\hat{\rho}_{2}, \hat{p}_{2}, \delta_{2}, P_{2}, H_{0_{2}}, \alpha_{2}\right)=\mathcal{J}\left(P_{\infty}, H_{0_{\infty}}, \alpha_{\infty}, \hat{\rho}_{d}, \beta_{2}\right), \\
& \left(\hat{\rho}_{1}, \hat{p}_{1}, \delta_{1}, P_{1}, H_{0_{1}}, \alpha_{1}\right)=\mathcal{J}\left(P_{\infty}, H_{0_{\infty}}, \alpha_{\infty}, \hat{\rho}_{d}, \beta_{1}\right), \\
& \left(\hat{\rho}_{3}, \hat{p}_{3}, \delta_{3}, P_{3}, H_{0_{3}}, \alpha_{3}\right)=\mathcal{J}\left(P_{1}, H_{0_{1}}, \alpha_{1}, \hat{\rho}_{d} / \hat{\rho}_{1}, \beta_{3}\right) .
\end{aligned}
$$

Note that here we have reverted to the subscripting scheme used for multiple shock wave interactions. Matching the pressure and flow deflection angle across the shear layer that originates at the intersection point we have

$$
\begin{aligned}
& \hat{p}_{2}=\hat{p}_{1} \hat{p}_{3}, \\
& \delta_{2}=\delta_{1}+\delta_{3} .
\end{aligned}
$$

Collectively, Eqs. (64), (65), (66), (67), and (68) represent 20 equations in the 20 variables $\hat{\rho}_{1,2,3}, \hat{p}_{1,2,3}, \delta_{1,2,3}, P_{1,2,3}$, $H_{0}, 2,3, \alpha_{1,2,3}, \beta_{2,3}$ and for convenience we choose $\beta_{1}, P_{\infty}$, $H_{0_{\infty}}, \alpha_{\infty}$, and $\hat{\rho}_{d}$ as parameters. For a given free stream condition and shock angle this system describes a path of solutions for each $H_{0_{\infty}}$ from which we may study the influence of real gas effects. The equations are solved using 

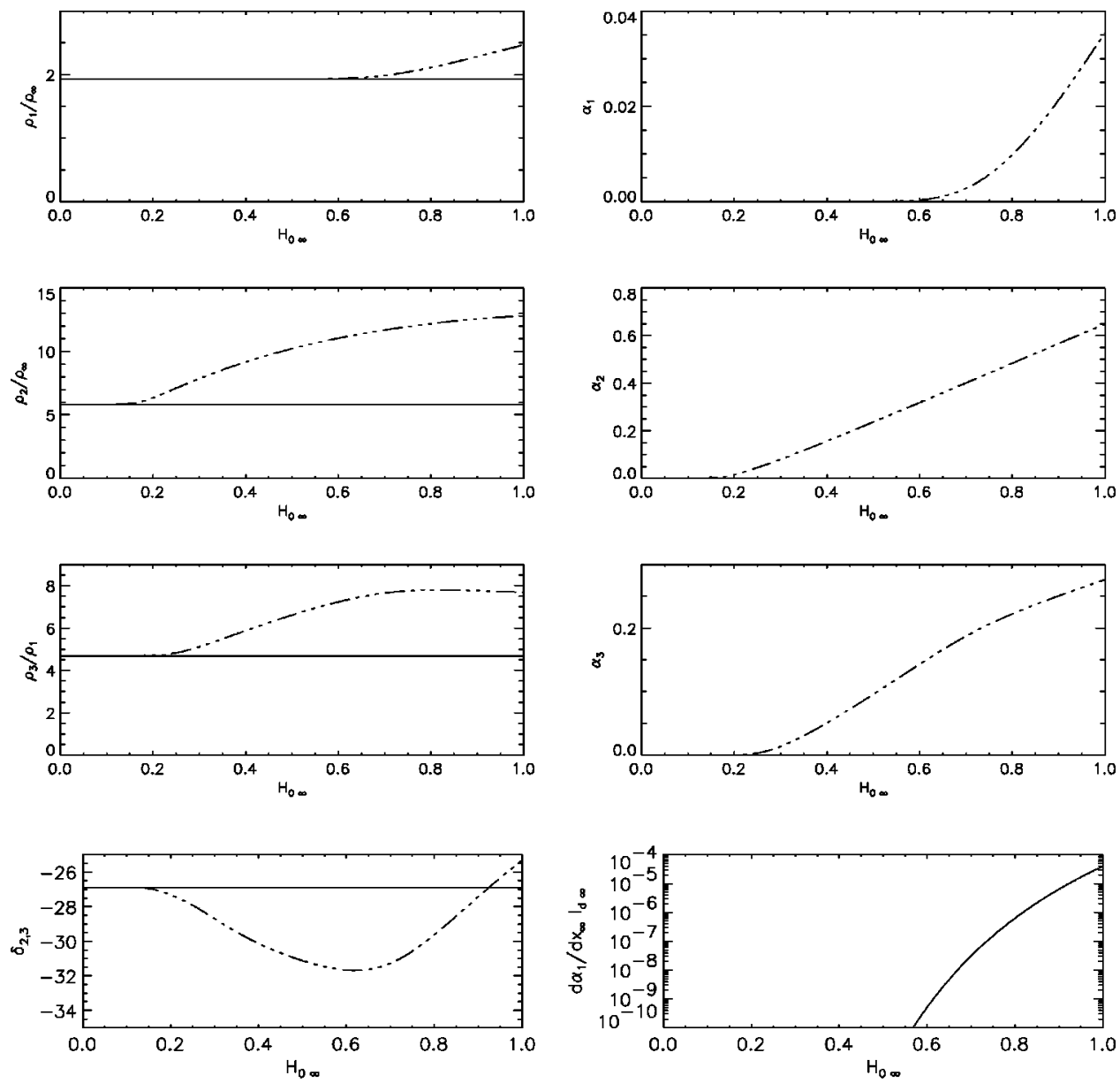

FIG. 9. Density variations, dissociation levels, shock wave angles, streamline deflection, and reaction rates as functions of stagnation enthalpy, $H_{0_{\infty}}$, at a $\lambda$-shock point in an ideal dissociating gas. The free-stream conditions are $P_{\infty}=0.025, \quad \hat{\rho}_{d_{\infty}}=1$ $\times 10^{7}, \alpha_{\infty}=0, \beta_{1}=16^{\circ}$. Solid curves are the frozen solutions. $-\cdots-$ are the equilibrium solutions.
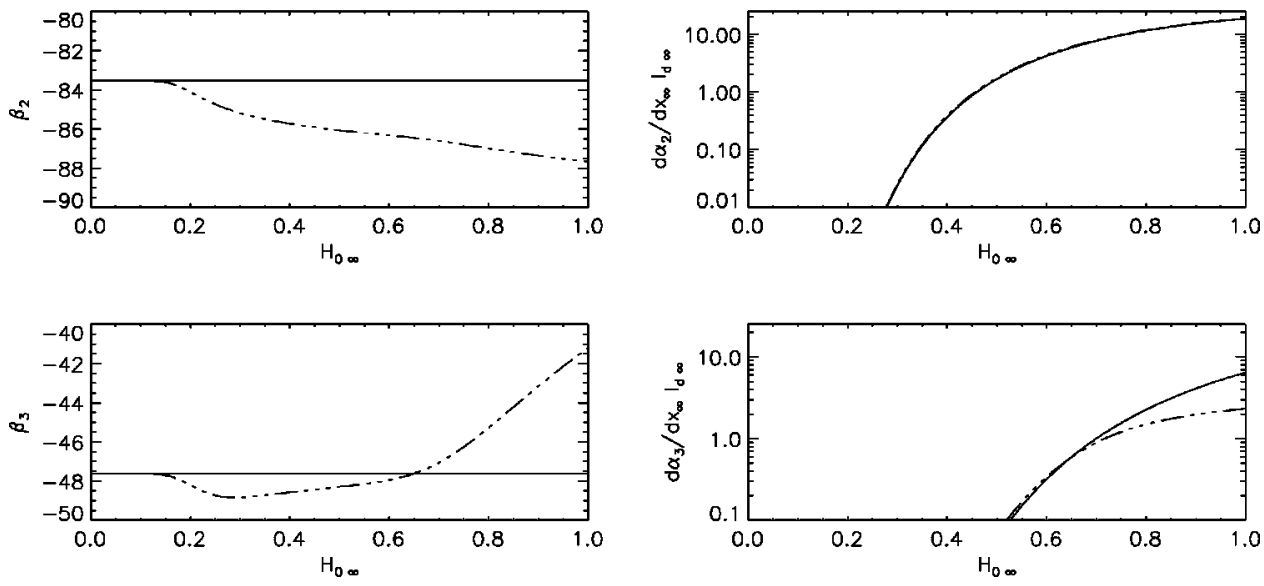

Newton's method for the cases of frozen and equilibrium chemistry and the solution is continued in $H_{0_{\infty}}$. Solution curves for the density, dissociation levels, shock wave angles, flow deflection angles, and reaction rates are plotted in Fig. 9. In performing the above computations, and in the interpretation of the solutions obtained, the value of the formalism laid out in the current paper becomes apparent.

An interpretation of the solutions that are illustrated in Fig. 9 follows from the results presented in Fig. 6. We see that the influence of real gas effects increases strongly with increasing stagnation enthalpy, $H_{0_{\infty}}$. Dissociation is negligible behind the weak impinging wave and dissociation lev- els increase proportionally for the moderate strength wave, (3), and the nearly normal wave, (2). The corresponding increases in the density are the most important real gas effects.

A further similarity with the conclusions drawn from Fig. 6 is the behavior of the normalized reaction rates. The reaction rate behind the impinging wave, $d \alpha_{1} / d \hat{x}_{\infty}$, is negligibly small in both the frozen and equilibrium limits. The angles of the stronger shock waves, $\beta_{2}$ and $\beta_{3}$, vary in the frozen and equilibrium limits and so produce different initial reaction rates. Behind shock (3) the initial reaction rate in the equilibrium limit is an order of magnitude smaller than that behind the nearly normal wave, (2). This is a significant ef- 


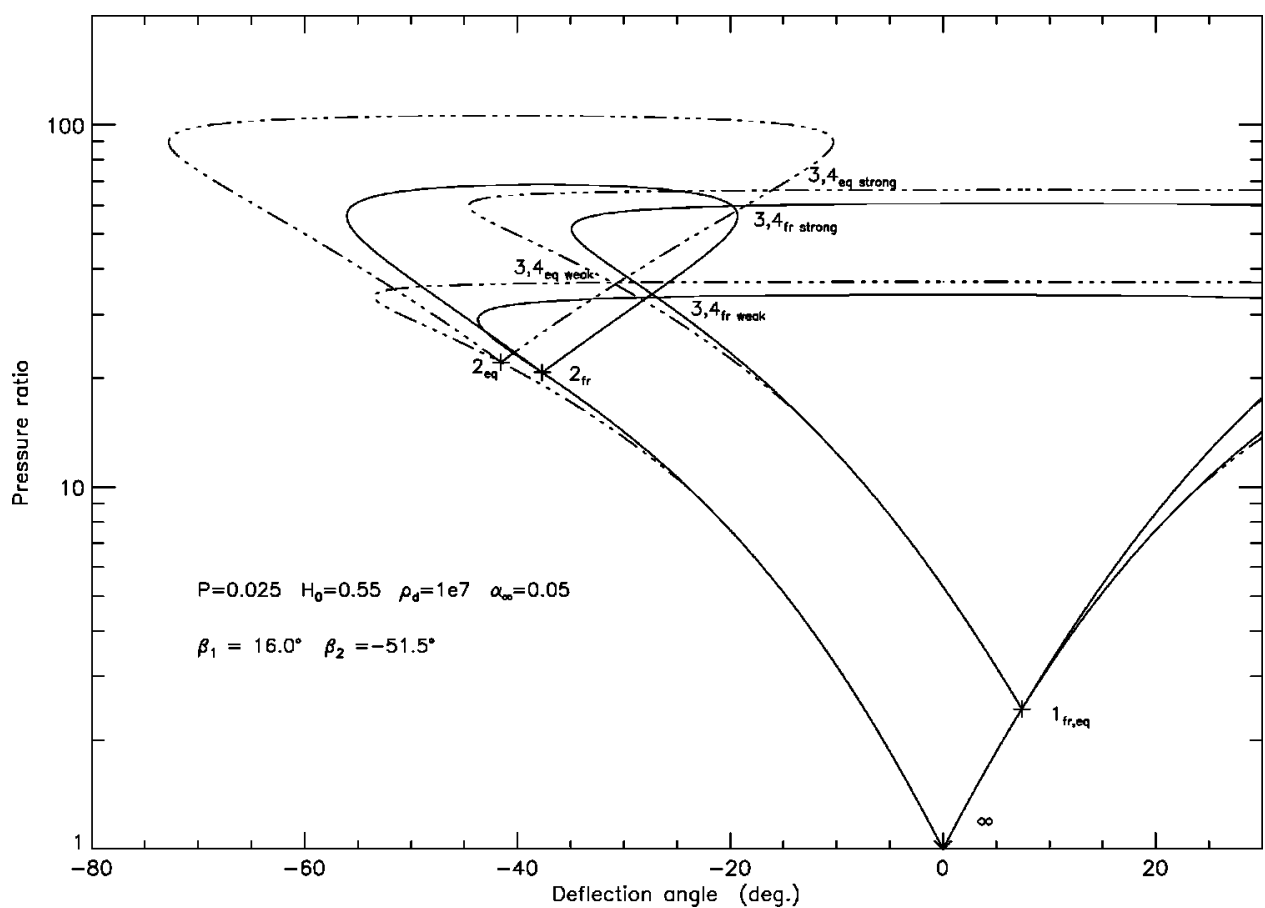

FIG. 10. The $p-\delta$ plane representation of the interaction of oblique shock waves in an ideal dissociating gas. The incident shock angles are $\beta_{1}=16^{\circ}$ and $\beta_{2}=-51.5^{\circ}$. The free stream conditions are $P=0.025, H_{0}=0.55, \hat{\rho}_{d}=1$ $\times 10^{7}, \alpha_{1}=0.05$. The shock interaction solution is represented graphically by the interaction of the loci for the reflected shock waves (3) and (4). Solid curves are the frozen shock loci. - $\cdots-$ are the equilibrium shock loci. fect because it controls the relaxation of the density downstream of the interaction.

The strongest effect on the measurable wave angles is observed in the deflection angle, $\delta_{2,3}=\delta_{2}=\delta_{1}+\delta_{3}$, of the shear layer generated at the impingement point. We see from the mapping of the streamlines into the $p$ - $\delta$ plane in Fig. 7 that the strongest effects on the flow deflection angle are apparent for nearly normal waves. This causes the strong initial effect on the flow deflection angle as $H_{0_{\infty}}$ increases. The effect reduces as the dissociation level behind shock (3) rises at still larger values of $H_{0_{\infty}}$.

Hornung $^{9}$ has studied the influence of real gas effects on the Mach reflection problem. Given the results discussed here, it is reasonable to expect significant real gas effects on the blunt body shock interaction problems studied by Edney ${ }^{10,11}$ The latter subject has been investigated experimentally by the authors (Sanderson ${ }^{12,13}$ ) and will form the basis for a forthcoming paper.

In contrast to the strong chemical nonequilibrium effects observed in the $\lambda$-pattern of shock waves, consider the asymmetrical interaction of two oblique shock waves in steady flow, as illustrated in Fig. 10. Readers skilled in the field of Mach reflection will note that the condition shown lies slightly beyond the von Neumann transition point for the case of frozen chemistry, and slightly before the von Neumann transition point for the case of equilibrium chemistry. Following the lead of Hornung and Smith ${ }^{7}$ and Hornung, Oertel, and Sandeman, ${ }^{9}$ this represents a flow field where transition occurs locally at the intersection point (frozen solution) with the global transition (equilibrium solution) occurring for stronger interacting waves. For shock angles lying between the frozen and equilibrium von Neumann transition points, the length scale of the resulting flow field is determined by the chemical kinetics, this being the only scale available at the intersection point.
Despite the curiosity of a local kinetic length scale in shock wave interaction problems, the difference between the frozen and equilibrium transition points noted above remains subtle. This is a consequence of the weakness of the waves up to the transition point, as discussed in Sec. III. The primary interest in these problems lies in the strong density gradients downstream of the strong shock waves due to nonequilibrium dissociation. In the case of self-similar, unsteady Mach reflection from a wedge, transition is thought to occur at the sonic point on the reflected shock locus. More significant effects of nonequilibrium thermochemistry on the transition points should therefore be expected since the shock waves are stronger at transition under the sonic criterion.

\section{CONCLUSIONS}

A compact dimensionless framework has been presented for the elucidation of real gas effects on normal, oblique and interacting shock waves. Emphasis has been placed on the use of a consistent set of nondimensional parameters throughout so that the results are directly usable in analyzing relaxing flows. Identities are given for alternate choices of parametrization. A simple form is given for the reaction rate parameter that relates the chemical and fluid time scales. Oblique shock jump conditions are presented for all of the dimensionless parameters. Shock wave interaction problems are considered by analyzing the structure of the sonic line and by mapping the problem in the pressure-flow deflection angle plane, in terms of the same consistent parametrization. It is shown that real gas effects are most pronounced as locally increased gas densities behind only the strongest portions of the shock waves present in the flow field. Real gas influences on Mach reflection transition points are therefore 
seen to be of secondary importance to real gas effects in the vicinity of the post-transition $\lambda$-shock configuration at the ends of the Mach stem.

${ }^{1}$ W. G. Vincenti and C. H. Kruger, Introduction to Physical Gas Dynamics (Krieger, Malabar, FL, 1965).

${ }^{2}$ M. J. Lighthill, "Dynamics of a dissociating gas. Part I. Equilibrium flow," J. Fluid Mech. 2, 1 (1957).

${ }^{3}$ N. C. Freeman, "Nonequilibrium flow of an ideal dissociating gas," J. Fluid Mech. 4, 407 (1958).

${ }^{4}$ J. G. Hall, A. A. Eschenroeder, and P. V. Marrone, "Blunt-nose inviscid airflows with coupled nonequilibrium processes," J. Aerosp. Sci. 29, 1038 (1962).

${ }^{5}$ R. Courant and K. O. Friedrichs, Supersonic Flow and Shock Waves (Interscience, New York, 1948).

${ }^{6} \mathrm{H}$. G. Hornung, "Regular and Mach reflection of shock waves," Annu. Rev. Fluid Mech. 18, 33 (1986).
${ }^{7}$ H. G. Hornung and G. H. Smith, "The influence of relaxation on shock detachment," J. Fluid Mech. 93, 225 (1979).

${ }^{8}$ M. Kaneshige and J. E. Shepherd, "Oblique detonation stabilized on a hypervelocity projectile," 26th Symposium (International) on Combustion, Napoli, Italy, 1996.

${ }^{9}$ H. G. Hornung, H. Oertel, and R. J. Sandeman, "Transition to Mach reflexion of shock waves in steady and pseudosteady flow with and without relaxation," J. Fluid Mech. 90, 541 (1979).

${ }^{10}$ B. E. Edney, "Anomalous heat transfer and pressure distributions on blunt bodies at hypersonic speeds in the presence of an impinging shock," FFA Report 115, 1968.

${ }^{11}$ B. E. Edney, "Effects of shock impingement on the heat transfer around blunt bodies," AIAA J. 6, 15 (1968).

${ }^{12}$ S. R. Sanderson, "Shock wave interaction in hypervelocity flow," Ph.D. thesis, Caltech, 1995.

${ }^{13}$ S. R. Sanderson and B. Sturtevant, "Shock impingement heating in hypervelocity flow," Proceedings of the 20th International Symposium on Shock Waves, Pasadena, 1995. 